\title{
AN EMPIRICAL STUDY OF WOLF-RAYET STELLAR WIND IONIZATION STRATIFICATION
}

\author{
MATTHEW J. DALTON, PAUL A. CROWTHER and \\ ALLAN J. WILLIS \\ Department of Physics and Astronomy, University College London \\ Gower Street, London WC1E 6BT, U.K.
}

\begin{abstract}
We present an analysis of high resolution UV (IUE) and optical (INT, AAT and ESO) spectroscopy of eight galactic WN and WC stars. We examine the correlation between ionization potential (IP) and the velocity structure of the stellar winds and find important correlations between line-width, IP and excitation potential (EP). These results provide potential constraints on the velocity law used in models of WR atmospheres.
\end{abstract}

Key words: stars: Wolf-Rayet - atmospheres - stratification - winds

\section{Introduction}

Beals (1929) first demonstrated the now familiar correlation between ionization potential (IP) and line-width for WR stars (Kuhi 1973). This view was questioned by Willis (1982), who argued that the correlation existed between excitation potential (EP) and line-width. Hillier (1989) reconciled the two opposing views, showing that the principal correlation is between IP and line-width, whilst a weaker but significant relationship exists between the IP and EP. Recently the line-width vs. EP correlation has been re-examined by Niedzielski (1994).

\section{Analysis and results}

We use high resolution $(\sim 0.1$ to $2 \AA)$ UV and optical observations to examine the velocity structure of eight galactic WR stars. For each star, a large sample of emission lines were identified in the rectified spectra, and measurements made of the FWHM by fitting Gaussian profiles. Where appropriate we corrected for the multiplet nature of individual line profiles. Quantitative comparisons of FWHM vs. IP were made for each star, and correlations assessed by determining linear least squares fits in each case, and Pearsons $r$ coefficient. Similar comparisons were made with FWHM vs. EP for the program WN 5-7 stars.

Our results show correlations between the FWHM and IP for each star, which are summarised in Table I. There does not appear to be a trend connecting the gradient $(g)$ or FWHM at zero IP $(k)$, with either spectral subtype, $v_{\text {black }}$ or $v_{\max }$. This lack of distinct correlation of FWHM with IP 
is not surprising when the variety of line formation mechanisms and range of optical depths of the lines measured in our sample are considered. This is supported by the relatively large errors on $g$ and $k$ which are due to the intrinsic scatter of the data resulting from stratification effects within each ionization stage. We find that the lower EP transitions are formed at higher velocities in three of the four WN 5-7 stars (WR6, WR136 and WR78), and may also be the case in the fourth, WR134. These findings support the results of Niedzielski (1994).

TABLE I

Summary of ionization stratification study

\begin{tabular}{|c|c|c|c|c|c|c|c|}
\hline \multicolumn{2}{|c|}{ star } & \multirow[t]{2}{*}{ subtype } & \multirow{2}{*}{$\begin{array}{c}g \\
\left(\mathrm{~km} \mathrm{~s}^{-1} \mathrm{eV}^{-1}\right)\end{array}$} & \multirow{2}{*}{$\begin{array}{c}k \\
\left(\mathrm{~km} \mathrm{~s}^{-1}\right)\end{array}$} & \multirow[t]{2}{*}{$-r$} & \multirow{2}{*}{$\begin{array}{r}v_{\max } \\
(\mathrm{km}\end{array}$} & \multirow{2}{*}{$\begin{array}{l}v_{\text {black }} \\
\left.\mathrm{s}^{-1}\right)\end{array}$} \\
\hline HD & WR & & & & & & \\
\hline 50896 & 6 & WN 5 & $-11.2 \pm 3.03$ & $2359.5 \pm 214.50$ & 0.605 & 2650 & 1700 \\
\hline 191765 & 134 & WN 6 & $-20.2 \pm 3.96$ & $3495.8 \pm 235.13$ & 0.816 & 2300 & 1905 \\
\hline 192163 & 136 & WN 6 & $-19.1 \pm 3.96$ & $2932.4 \pm 239.94$ & 0.790 & 2000 & 1605 \\
\hline 151932 & 78 & WN 7 & $-6.6 \pm 2.00$ & $760.1 \pm 115.91$ & 0.613 & 2150 & 1365 \\
\hline 96548 & 40 & WN 8 & $-5.5 \pm 1.49$ & $970.1 \pm 55.39$ & 0.742 & 1800 & 930 \\
\hline 165763 & 111 & WC 5 & $-9.9 \pm 2.23$ & $2717.3 \pm 184.85$ & 0.686 & 3550 & 2145 \\
\hline 192103 & 135 & WC 8 & $-18.7 \pm 4.12$ & $2381.5 \pm 242.17$ & 0.687 & 1900 & 1405 \\
\hline 164270 & 103 & WC 9 & $-14.6 \pm 3.02$ & $1597.9 \pm 137.14$ & 0.667 & 1400 & 1190 \\
\hline
\end{tabular}

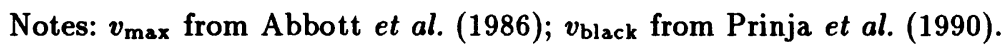

The predictions of ionization and excitation stratification made by the standard model, (e.g., Hillier 1989), have been confirmed observationally once more by our analysis. Our dataset provides a potential method of constraining the velocity laws of WR stars. We hope to provide such constraints in future work. The information available from stratification effects, are certain to play a crucial role in the development of our knowledge of wind dynamics and variability of the WR stars.

\section{References}

Abbott, D.C., Bieging, J.H., Churchwell, E., Torres, A.V. 1986, ApJ 303, 239

Beals, C.S. 1929, MNRAS 90, 202

Hillier, D.J. 1989, ApJ 347, 392

Kuhi, L.V. 1973, in: M.K.V. Bappu \& J. Sahade (eds), Wolf-Rayet Stars and High Temperature Stars, Proc. IAU Symp. No. 49, (Dordrecht: Reidel), p. 205

Niedzielski, A. 1994, $A \& A$ 282, 529

Prinja, R.K., Barlow, M.J., Howarth, I.D. 1990, ApJ 361, 607

Willis, A.J. 1982, MNRAS 198, 897 